\title{
Academic specialist education in headache medicine. Next move for the headache community
}

The headache community is experiencing a period of new cultural identity worldwide. The basic research applied to headache disorders, always alive and flourishing, has reached heights of absolute excellence in the last few years, also by producing an extraordinary induction in terms of new and efficacious therapeutic opportunities $[1,2]$. Unfortunately, a gap has always existed between this innovative scientific know-how and its translation into educational ventures. In addition, most training initiatives in the headache field are supplied in geographical areas already presenting high medical standards regarding headache disorders. It easy to understand how the gap between adequate clinical management of these disorders and the average training level of the local medical body becomes increasingly wide. The sequel is a void impossible to fill for all those countries which begin to express cultural interest towards headache medicine. At the same time, an intermediate area exists between the countries of the first group and the second one, which includes a wide multidisciplinary body of physicians who, although faced with headache clinical dilemmas, do not receive adequate updating of the relative expertise.

Headache medicine training appears to be the next compelling step towards spreading a new medical culture regarding this area. Not many universities in Europe or America present in their integrated courses formative credits for headache teaching. Ante lauream education is lacking if compared to the clinical expertise demanded from the medical profession. This difference between the physician's daily impact with headache and education is still huge. Academic institutions, where headache research resides, must necessarily transfer this knowledge to educational activities. As a logical consequence of these considerations, there is a need for headache training provided through independent highprofile tools with extremely updated programmes.

We all know the diagnosis of headache disorders does not demand special investigations but only special skills. This represents one of the hurdles towards the effective care of headache disorders indicated by the World Health Organization (WHO) in the Global Campaign (GC) to Reduce the Burden of Headache Worldwide, or shortly, Global Campaign against Headache [3]. These skills are pointed out as issues to be inserted in the educational course completion of the medical profession. "Education, as the key of effective headache management, ... should begin in medical schools by giving headache a place in the undergraduate curriculum..." [4]. 
This is, among others, the pillar in the strategic priorities to be achieved to obtain a substantial reduction in the burden of headache. The topic is not new, although still unsolved [5].

Post lauream academic programmes dedicated to headache are beginning to appear [6], and the spread of similar university training courses worldwide will bridge the gap existing between extreme research qualification and correct clinical management. In this light, within the GC we will move towards a high-profile headache expertise broadcasting in different areas worldwide. Yet locally, these specialists will be able to provide back up and headache skills to primary care doctors.
Our journal aims to stimulate a virtual forum debating this core passage for the headache community, from research acquisitions to their translation into headache management.

The GC will place Specialist Education among its priorities in this new headache era. We need to sow, with the aid of innovative, interactive and modern didactic modalities, new and fertile grounds with headache medicine expertise. Distance learning on demand could perpetuate, after academic formation, specialists' updating in order to incorporate headache education in their clinical daily style [7].

\section{Paolo Martelletti} Editor-in-Chief

\section{References}

1. Lipton RB, Bigal M (2005) Headache: triumphs in translational research. Lancet Neurol 4:11-12

2. Geppetti P, Capone JG, Trevisani M, Nicoletti P, Zagli G, Tola MR (2005) CGRP and migraine: neurogenic inflammation revisited. J Headache Pain 6:61-70

3. Steiner TJ (2004) Lifting the burden: the global campaign against headache. Lancet Neurol 3:204-205
4. WHO (2004) Headache Disorders. World Health Organization, Fact sheet No. 277.

http://who.int/mediacentre/factsheets/fs 277/en/

5. WHO (2000) Headache Disorders and Public Health. Education and Management Implications. WHO/MSD/MBD/00.9. pp 1-8
6. Master in Headache Medicine (2005) 2nd School of Medicine. University of Rome La Sapienza. http://w3.uniroma1.it/headache/

7. Martelletti P (2002) The distance learning on headache disorders and pain. J Headache Pain 3:159 